\title{
The consultant practitioner- an evolving role to meet the changing NHS
}

Abstract

Introduction: Since the introduction of the consultant practitioner role and huge variability in purpose and context, the role has had to evolve in response to the changing needs of the $\mathrm{NHS}$ to achieve sustainability and transformation of services.

Aim: This paper aims to present a reflection on the current relevance of the consultant practitioner role and the impact of an action learning set in hastening its evolution in one NHS Foundation Trust.

Methodology: From a process of collective critical reflection on their practice, six consultant practitioners analysed the impact they have had on influencing services and empowerment of their patients. Additionally, they have analysed the impact of an externally facilitated action learning set as a catalyst for change.

Findings: All six consultant practitioners recognised that by working together through the learning set enabled them to be more influential and effective. It encouraged them to share their experiences of continuous service improvement and crystalised their views on the impact they have had in delivering the organisation's vision.

Conclusion: From their critical reflection, six consultant practitioners acknowledge the influence of the action learning set to accelerate their confidence and competence to lead, and evaluate new models of care delivery at scale and pace. They recognised how far they have travelled in achieving the four dimensions of the role and ultimately their impact on their local Sustainability and Transformation Plan and organisation's vision. Through their reflection, they resolved to keep abreast of research and policy developments to enable services to remain flexible and responsive to need.

Words: 253 


\section{Introduction}

Since the introduction of what were first termed Non-medical consultant roles in 2000 (DOH 1999) there has been a deal of discussion around the effectiveness and appropriateness of the roles (Hayes \& Harrison 2004, Guest et al 2004). Initially the consultant nurse role was developed in response to a poor career structure for senior clinical nurses and concerns regarding patient safety. In particular it was proposed that this new tier would strengthen nursing leadership and retain experienced nurses in practice (DH 1999). It was envisioned that the 'super nurse' role would not only improve the patient experience but would also act as a role model for junior nurses to aspire to. Following on from the rise of the nurse consultant role, the Department of Health (2000) published its vision for the development of consultant therapists. This role was designed to distinguish allied health professions from their medical and nursing counterparts and aimed at the full range of allied health professionals to enhance patient outcomes, provide career progression and strengthen professional leadership. For simplicity this paper will use the term 'consultant practitioner' when referring to both nursing and allied health professional consultants. This paper presents a reflection on the current relevance of the consultant practitioner from the perspective of the authors (five consultant nurses and one consultant physiotherapist) and examines how the introduction of an action learning set impacted their role and hastened its evolution to meet the demands of the NHS in 2017.

\section{Background}

The current challenges facing the NHS are well known - ever increasing demand on the health and social care systems not being matched by a related increase in funding, coupled with a static working age population and the consequent impact on the ability to recruit staff to deal with the increased demand (NHS 2014). To address these challenges NHS England published the Five Year Forward View (2014), and subsequently invested in Vanguard project sites across the UK to develop new models of care with a greater focus on prevention, public health, ownership of own care, sustainability and reducing barriers between providers. In spite of the advances the NHS has made over the two years since its publication, evidenced by the Next Steps in the NHS Five Year Forward View (2017), the authors believe that the modern consultant practitioner offers a key leadership role in the delivery of these changes to take forward the NHS in this critical new direction.

Despite huge variability in purpose and context, the consultant practitioner roles were introduced around 4 core functions: i) expert clinical practice; ii) leadership and consultancy; iii) education, training and development iv) research and service development (DH 1999). After some years in role, the authors are now interpreting and delivering the core functions of the consultant practitioner role in a transformational and evolutionary way in their own NHS Foundation Trust and disseminating their work through networking nationally and internationally (Rosser et al 2017). Whilst the key themes of the current role still mirror the original, the focus has been channelled in response to the changing needs of the local health and social care economy and to the ongoing policy changes impacting on the further development of the National Health Service (NHS) (NHS 2014, 2017). The new intuitive response to achieve sustainability requires innovation, forward thinking and to some degree risk taking as identified by the NHS (2017). 
At the local level, the postholders were employed to work as clinical leaders, and not specifically as managers of services responsible for budgetary control. The four dimensions of the consultant role were embedded as a requirement with the express purpose to influence change through clinical knowledge. It was expected that the consultant practitioner role would have a much wider understanding of the organisational impact, service development and cross boundary working with other professional groups at a senior level than for example, the clinical nurse specialist. The greatest difference between the two roles (specialist and consultant) relates to their leadership and their level of accountability, which for the consultants rests clearly with themselves. At this local level, the Board of Governors of the organisation firmly expected the consultant practitioners to operate at the highest strategic level within their area of expertise and they respected highly their views regarding change management to improve the organisational direction. Additionally, whilst the post holders can be considered expensive in terms of staff costs they are believed by the Director of Nursing to be cost effective. Many hold clinics, lead on CQUINS such as frailty or dementia, which are income generating and many introduce new models for working which are not only efficient in their ways of working but cost saving (Ryan 2016). The authors draw on their experiences of working for an integrated primary and acute care Vanguard site to illustrate the evolution of the consultant practitioner role. The current role

The authors are all employed by an acute NHS Foundation Trust that serves a predominantly rural health and social care community of some 200,000 people with a high proportion of residents aged over 65 (21.6\%) (Yeovil Hospital Healthcare 2017). As a Vanguard site, the acute trust and other NHS partners are working together with Social Care to establish an Accountable Care Organisation responsible for improving population health through a suite of integrated care models, a person-centred approach and community empowerment. The vision is 'Leading innovation and integration for a healthier, more independent population' and has three key objectives. To achieve:

- Informed people empowered to take responsibility for their health and wellbeing.

- An ambitious and adaptive workforce, working creatively to deliver exceptional care.

- A seamless, integrated and responsive network of care services, working together to do the right thing for patients.

(Balian \& Higman 2017)

The two core integrated models implemented to date are i) complex care and ii) enhanced primary care. Taking the lead from the Five Year Forward View (2014), complex care targets the $1.5 \%$ of the population with the most complex and intense needs for support. Enhanced primary care focuses on enabling practices to move from a reactive to a proactive approach, focussing on improving the health of their community and ensuring a sustainable model of general practice where GPs work as part of a wider team. The next areas under development include iii) systematised surgery and iv) a new approach to urgent care delivery.

\section{Creating and Leading Change}


Given several years in role and engaged in reflective discussion, we have each shaped our role to meet the emerging needs of the local community and we have been instrumental in influencing the service we lead towards empowering our patient group, focusing where appropriate on efficiencies but for the most part influencing change. The following sections review the impact we have had on our services.

\section{Expert clinical practice}

The key component of the $1^{\text {st }}$ core function of our role is that of expert clinical practice, which we, the authors all demonstrate to a high level in our individual specialities (stroke, infection control and tissue viability, emergency care, respiratory, integrated care and rehabilitation and Dementia and Care of Older People). We believe that working in a clinical capacity for a significant period of the working week enhances the consultant practitioner role, enables us to directly influence the patient pathway management, offers the opportunity to act as role models to other specialist practitioners and collaborate and work interprofessionally with medical and other consultant practitioners to shape the service and patient experience. Although this $50 \%$ of the role in expert clinical practice has changed little since it was first envisaged, we believe it is an essential function of the role. Examples of expert practice include nurse prescribing, nurse-led clinics and the development of out-reach teams such as early supported discharge teams to enable timely discharge home for patients after their stroke enabling specialist rehabilitation in the home setting.

\section{Leadership and consultancy}

The $2^{\text {nd }}$ core function of leadership and consultancy has a strong focus on transformational change ensuring that the post holders not only implement change but bring the team along with them. Building functional and attentive teams that become highly effective is illustrated in the NHS (2014) publication of Building and Strengthening Leadership: Leading with Compassion, the work of Cummings and Bennett (2012), implementing plans for Compassion in Practice. Cummings and Bennett (2012) acknowledge the characteristics of dysfunctional teams and recognise the importance of team leadership and reflexivity by the team to learn where adjustments should be made. Consultancy is a diverse concept but was summarised by Caplan's model (Manley 1997) over 40 years ago as having 4 key types - client centred, consultee centred, programme centred and organisational centred. One example from our own practice by the Consultant Nurse for stroke is being part of a national body for the management of stroke patients which influences stroke care at a national level. Working at that level facilitates enhanced knowledge and awareness of the national strategy such as the National Institute for Health and Care Excellence (NICE), Royal College of Physicians Stroke guidelines and Sentinel Stroke National Audit Programme (SSNAP) which culminated in the significant success of retaining stroke services locally.

\section{Education, training and development}

Education, training and development is the $3^{\text {rd }}$ core function of the consultant practitioner. From the initial vision (DH 1999), the NHS Executive clearly expected the consultant practitioner to have an active role in educating others to ensure a practical application of theory into clinical practice. As the role has the important clinical element it demands that consultant practitioners' clinical knowledge is up to date and so can be reflected in the 
education process. This is achieved not only through day to day exposure to the 'real' situation but importantly with reading, networking, attendance at regional and national meetings and the ability to discuss complex cases with senior colleagues, keeps the consultant practitioner abreast of the most up-to-date evidence related to their scope of practice. A significant advantage of these external meetings may include an early awareness and sharing of any changes in policy and practice enabling the organisation to be more prepared. This networking and education arm of the role, sets the role apart from clinical nurse specialists who whilst they have good clinical credibility the role does not typically extend this widely. Consultant practitioners have time within their job descriptions as it is accepted to be an important part of the role. Whilst there may have been some initial apprehension from medical colleagues this is no longer the case, certainly in our own organisation, at all levels, though as yet not all medical staff, now actively seek support and guidance from the consultant practitioners and we actively work in collaboration. They respect the consultant practitioner's decision making having observed excellent outcomes for the patients based on current credible knowledge. The consultant practitioner team deliver extensive teaching programmes to patients, nurses and all grades of allied health care professionals. Many consultant practitioner roles also have honorary or substantive status with local universities and this has the additional benefit of promoting the role and showing an alternative career structure at an early stage to a wide audience of clinical staff. These roles provide 'real world' examples to purely academic staff which they consider to be invaluable. This is further evidence of the uniqueness of the role as we have multifaceted elements to our working week. In fact, since the original vision (DH 1999), the consultant practitioner role now requires full Masters level study, and for some, doctoral completion and, in itself demonstrates higher level critical thinking which offers in addition, credibility with academic colleagues.

\section{Research and service development}

The final consultant practitioner core function is research and service development and this is considered an essential aspect of the role. Research can consist of being Good Clinical Practice (GCP) trained and working on national or international studies or can be considered as working on locally developed audits. Both are equally valid and essential and from our experience, help consultant practitioners retain their level of critical thinking to shape strategic direction, based on available evidence. Indeed, Cummings and Bennett (2012) acknowledge the frustration of the often slow pace of change in the NHS. However, they also recognise from the work of Kotter (1995) that large-scale change can take years to effect, going through a number of stages to be successful. Key to success for service development, audit and evaluation is the effective team functioning as well as the seamless multi-professional working (Cummings and Bennett 2012). Consultant practitioners play a key role in both leading teams and establishing, and participating in, forums and networks to move patient care forward strategically and collectively. The fluid nature of the consultant practitioner role along with the confidence, expertise and experience gained from being fluent in the latest evidence-base and involved in advances in the specialist field, supports innovative service changes. For instance, an example from our own practice includes being the first organisation in the region to introduce the Ultra- $V$ decontamination system, alongside TECcare's high level disinfection technology, to provide a bleach free 
decontamination process, resulting in low levels of Health Care Associated Infections ( $\mathrm{HCAl})$. This is looking at new technology in a robust manner and implementing an evidence based change, which in turn improves patient safety and experience.

\section{The catalyst for change}

The evolution of the consultant practitioner role for ourselves was accelerated by participation in an externally facilitated action learning set (Rosser et al 2017). This was introduced as a result of a casual meeting between a senior educationalist at our local university and the Director of Nursing in our Trust and continued for 3 years. Prior to participating in the action learning set each of our focus was limited to being localised and insular. Following the work of the action learning set which coincided with the recent changes in the direction and priorities of the NHS, the consultant practitioners started to work more collaboratively making them more influential and effective (Rosser et al 2017). This work extending across health care boundaries had benefits for patients and working relationships with colleagues and for the organisation. One example of this collaborative approach was to promote improved experience for patients who have self-harmed. The consultant nurse in the Emergency Department collaborated with the local mental health services in developing an outreach programme of wound care education for mental health care workers which reduced the need for the patients to attend the Emergency Department as the wounds can be managed within their own service and environment which reduces the distress for the patient. Another example of consultant practitioners working together across specialties was the design and implementation of a dementia support role within the Emergency Department as it was identified that the care and management of patients with dementia in the busy environment of the Emergency Department did not support their needs appropriately. This role is the subject of ongoing evaluation which has demonstrated a positive impact on both the experience of patients with a cognitive impairment and their families when attending the department. It has enabled the facilitation of assessment and investigations required by these patients in an effective and therapeutic way, minimising distressed behaviour and ensuring that any interventions are provided in an appropriately skilled person-centred way. This initiative has achieved national acclaim (Valentine 2017). The action learning set also encouraged us to share our experiences of continuous service improvement and seek to collectively improve our practice, especially during this time of unprecedented financial pressure. The learning set crystallised our own views of the impact we had in providing clinical leadership, enabling teamworking and encouraging engagement at all levels to sustain and transform care (Ham et al 2016). Having an honest awareness of the daily pressures of the clinical services at the same time as leading from the front, enabled us to influence and more importantly deliver the organisation's vision (Rosser et al 2017). In spite of conflicting pressures within the organisation, this distinctive dual appreciation was invaluable and has resulted in each of us making significant change to our services that have made a profound difference to patient care .

Three further examples of the outcome of our new found collaboration includes the Ambulatory Emergency Care Service (AEC) that has been expanded and relocated in an area that the organisation previously used at times of bed capacity escalation. This loss of 7 flexible escalation beds was a great concern to the hospital board but the persuasive skills of one of us and evidence based from a smaller scale service delivery of AEC gave the board 
confidence to proceed. This change has enabled considerable admission avoidance and has also has had a positive impact on patient flow in the emergency department with improvement in achieving the 4 hour standard and more importantly patient satisfaction. A second example is the consultant nurse for tissue viability who identified an opportunity to reduce length of stay for patients with complex wound needs. Having insight and ground level knowledge led to the development of a new patient pathway facilitating early discharge. The third is the introduction of respiratory hot clinics (Royal College of Physicians 2017) to enable timely intervention by specialists to prevent admission of complex or rapidly deteriorating patients.

Strategically, an important outcome of the action learning set was revisiting our role in relation to integrating the core functions. We recognised at our monthly meetings of the need to recognise just how we were achieving in each of the four functions. We acknowledged that the process of integration was intuitive and, especially when introducing a new service, required the introduction of a new training programme, new protocols developed, implemented and evaluated. We learned considerably from each other. An example of integrated cross functional working is the consultant physiotherapist who drew on a firm foundation of research, teaching and leadership to lead the introduction, development and training of support workers as health coaches (Band 3) and keyworkers (Band 4) who support people to manage their own health, coordinate care and deal with non-medical issues. In addition the health coaches work with the wider community to deliver public health initiatives, such as physical activity or healthy eating. Her leadership in innovating care using her expert practice and education functions, has revolutionised the service and she will be auditing and publishing the results.

Working collaboratively as a result of the action learning set has allowed us to reflect on our many successes. We have recognised the impact we have had in uniquely bridging between the worlds of clinical care and strategic management and planning and positioning ourselves to lead effective implementation through influencing appropriate service change. There has been a shifting of focus from working within an individual organisation to greater integration and cross boundary working. This joint working has now extended to include working with other organisations within the health and social care economy including voluntary sector organisations, commissioners and public health. As consultant practitioners we are well placed to ensure that all provider organisations are working towards achieving Best Practice Tariffs (NHS 2016) to deliver excellence in patient care. Our consultant practitioners' innovative roles also enable us to be influential in service planning at both commissioning and delivery levels.

\section{Conclusion}

Following three years of engaging collectively in an action learning set, we have reflected on all of our achievements. While recognising the challenges that this learning set presented to us at the outset, given we knew little of each other or our ways of working, we have now developed a safe environment where we can meet and test out our ideas and celebrate our successes. There are of course times when we share our challenges and we now feel supported to seek our own solutions. From the Director of Nursing perspective (Ryan 2017), the organisation has noticed a change within the consultant practitioner group since the 
introduction of the ALS as they are much more visible within the organisation, they are seen as a team, they inspire each other professionally, such as producing research or publishing work and they are considered key contributors to the Trusts aims. Nevertheless, it is essential to continue to revisit the consultant practitioner role, as there is a high degree of clinical confidence in the team. The consultant practitioner is often the pinnacle position for many staff but for some it is the step to a Director post. This requires Trust-wide working with an excellent understanding of the clinical challenges including patient safety and risk which is what was designed for this role at the outset (Ryan 2017).

We conclude that in our experience, the consultant practitioner role has been critical in the context of a rapidly changing NHS where evolution of service delivery has been devolved to a local level. From our own experience, we were given the freedom and autonomy to explore and develop new models of care delivery, bringing integration out of the textbook and into reality. This has involved the design of new systems, new processes and new IT solutions that give visibility to a patient's care pathway across all their health care providers (Ham et al 2016). Services that used to be limited to those following an inpatient admission are now accessible at a much earlier point in a patient's pathway thereby preventing admissions. We have illustrated a number of projects where we believe we have made an impact. We believe that, triggered by the action learning set, we have maximised our learning from developing the new models of care to influence the Sustainability and Transformation Plan locally (Ham et al 2017). Together, our collaboration has helped us develop the confidence and competence to lead and evaluate new models of care at scale and pace, and we will continue to keep abreast of research findings and policy developments to enable services to remain flexible and responsive.

Words 3624

Balian B., and Higman, J. (2017) Symphony Integrated Healthcare: Person-centred, Coordinated Care: a new vision for Somerset lead by South Somerset PACS Vanguard. Presentation to the Symphony NHSE Medical Directorate Conference 7.6.17.

Cummings, J., Bennett, V. (2012) Compassion in Practice: nursing, midwifery and care staff - our vision and strategy. NHS Commissioning Board. Accessed at htt://www.england.nhs.uk//wp-content/uploads/2012/12/compassion-in-practice.pdf on 28.4.17

Department of Health (DH) (1999) Nurse, midwife and health visitor consultants: establishing posts and making appointments Health Service Circular HSC 1999/217. 29 September Leeds: NHSE

Department of Health (2000) Meeting the Challenge: A Strategy for the Allied Health Professions. November. Department of Health, London.

Guest, D.E., Peccei,R, Rosenthal, P., Redfern, S., Wilson-Barnett, J., Dewe, P...Sudbury, A. (2004) An evaluation of the impact of the nurse, midwife and health visitor consultants. Kings College, London 
Ham, C., Berwick, D., Dixon, J. (2016) Improving quality in the English NHS: A strategy for action. The Kings Fund, London. Accessed at:

https://webcache.googleusercontent.com/search?q=cache:UAl8TepFolAJ:https://www.kingsf und.org.uk/publications/quality-improvement $+\& \mathrm{~cd}=1 \& \mathrm{hl}=\mathrm{en} \& \mathrm{ct}=\mathrm{clnk} \& \mathrm{gl}=\mathrm{uk}$ on 28.4.17

Ham, C. Alderwick, H, Dunn, P. and McKenna, H. (2017) Delivering sustainability and transformation plans: From ambitious proposals to credible plans. 21 February. The Kings Fund, London. Accessed at https://www.kingsfund.org.uk/publications/deliveringsustainability-and-transformation-plans?gclid=CJTd9aLGx9MCFRdmGwodliOMcQ on 28.4.17

Hayes, J. and Harrison, A. (2004) Consultant nurses in mental health; a discussion of the historical and policy context of the role. Journal of Psychiatric and Mental Health Nursing. 11, 185-188.

Kotter, J.P.(1995) Leading change: why transformation efforts fail. Harvard Business Review, 73(2), 59-67.

Manley, K. (1997) A conceptual framework for advanced practice: an action research project operationalizing an advanced practitioner/consultant nurse role. Journal of Clinical Nursing, 6(3), p179 - 190. DOI: 10.1111/j.1365-2702.1997.tb00303.x

NHS (2014) Five Year Forward View. October. Accessed at: https://www.england.nhs.uk/publication/nhs-five-year-forward-view/ on 28.4.17.

NHS (2016) Additional Information: Best Practice Tariff Proposals. 29 July. Accessed at: https://www.gov.uk/government/news/update-to-best-practice-tariffs on 28.4.17

NHS (2017) Next steps on the NHS Five Year Forward View. March, Accessed at: https://www.england.nhs.uk/publication/next-steps-on-the-nhs-five-year-forward-view/ on 28.4.17

Rosser, E., Grey, R., Neal, D., Reeve J., Neal D., Smith, C. and Valentine J. (2017) Supporting clinical leadership through action: The nurse consultant role. Journal of Clinical Nursing, April 10, doi: 10.1111/jocn.13830.

Royal College of Physicians (2017) Integrated respiratory action network for patients with COPD. Future Hospital Programme, Royal College of Physicians, London. Accessed at https://webcache.googleusercontent.com/search?q=cache:BLGdYttsUBsJ:https://www.rcplo ndon.ac.uk/file/5602/download\%3Ftoken\%3D6mpONsdF+\&cd=10\&hl=en\&ct=clnk\&gl=uk on 28.4.17

Ryan, H. (2017) Personal communication with the Director of Nursing.

Valentine, J. (2017) Personal communication.

Yeovil Hospital Healthcare (2017) Yeovil District Hospital NHS Foundation Trust Operational Plan 2017/18 - 2018/19. Yeovil Hospital Healthcare. 
